\title{
Compressive Sensing Imaging with a Graphene Modulator at THz Frequency in Transmission Mode
}

\author{
V. A. Özkan*, T. Takan*, N. Kakenov ${ }^{\dagger}$, C. Kocabaş ${ }^{\dagger}$ and H. Altan* \\ *Department of Physics, Middle East Technical University, Turkey \\ Email: vedataliozkan@gmail.com \\ ${ }^{\dagger}$ Department of Physics, Bilkent University, Turkey
}

\begin{abstract}
In this study we demonstrate compressive sensing imaging with a unique graphene based optoelectronic device which allows us to modulate the $\mathrm{THz}$ field through an array of columns or rows distributed throughout its face.
\end{abstract}

\section{INTRODUCTION AND BACKGROUND}

$\mathbf{C}$ OMPRESSIVE sensing imaging techniques are proving useful in many applications, maybe more so than any other application $\mathrm{THz}$ imaging could have the most to gain since array imaging techniques are still not widespread. By discretizing the object using manually controllable pixels either near the object or on the image plane this method has been shown to effectively generate images of objects using single pixel $\mathrm{THz}$ detectors [1]. Here we demonstrate this with a unique graphene based optoelectronic device which allows us to modulate the $\mathrm{THz}$ field through an array of columns or rows distributed throughout its face.

The device uses a layered structure whereby the transmission of the THz-field is attenuated due to the large charge accumulation under applied differential bias between two sheets of graphene separated by an ionic conducting dielectric medium. Different regions of the device are isolated in sections of columns or rows which can be biased individually. The large charge build-up in each column or row can reduce the transmission by more than $20 \%$ which is sufficient to apply compressive sensing reconstruction techniques [2].

The set-up shown in Fig. 1 is used to demonstrate that a highly reflective, metal negative surface can be imaged. To reconstruct the image Total Variation (TV) minimization algorithm is used [3]. To assess the performance of the algorithm the reconstructed image was compared to the simulation of the ideal target. The comparison was done using the $\chi^{2}$ image quality assessment (IQA) index.

The graphene modulator changes rapidly from one pattern to another, making it faster than conventionally used masks [4]. Since the device can be manipulated as rows and columns instead of pixels the reconstruction performance is expected to decrease. The aim of this study is to analyze how the performance is affected.

\section{REsults}

Prior to experiments, the simulations were done to analyze image reconstruction. In Fig. 2, images obtained in simulation for number of masks given in horizontal axis are compared with the original image and the difference between them is

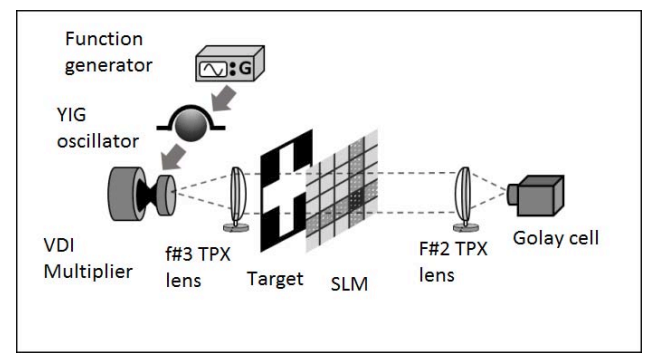

Fig. 1. THz signal is obtained from an yttrium iron garnet oscillators (YIG oscillator) output, multiplied by VDI Schottky diodes to $300 \mathrm{GHz}$. Then an F\# 3, TPX lens with $76.2 \mathrm{~mm}$ diameter collimates the light. The spatial light modulator and the target are close to each other and to the lenses to minimize optical sources of error. Finally an F\# 2 TPX lens with $76.2 \mathrm{~mm}$ diameter focuses the light on receiver, a Golay cell. The target is an aluminum plate with a plus sign cut out.

plotted on the vertical axis. The simulations considered the $16 \times 16$ patterns which were used in the experiment. In the simulations, the image is obtained using less than half the number of masks required for deterministic reconstruction algorithms.

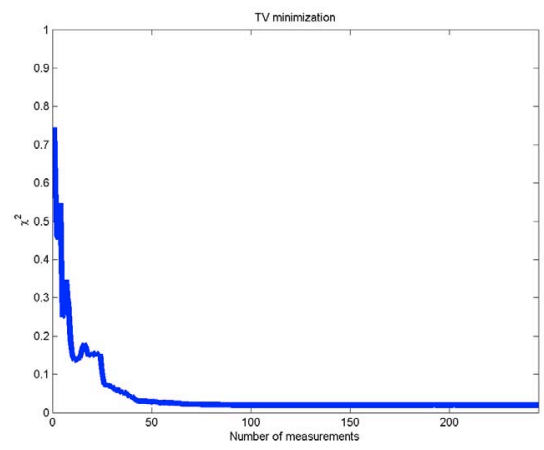

Fig. 2. Simulation result for the TV reconstruction algorithm. The target is a negative perfectly reflective plus sign and all optical errors are ignored. Reconstruction uses logarithmic barrier and minimizes the total variations square.

\section{CONCLUSION}

A graphene SLM was used to reconstruct a negative metal target's image in transmission mode. The obtained image has a resolution of $16 \times 16$ pixels. In reconstruction TV minimization algorithm was used. 


\section{ACKNOWLEDGMENT}

This work was supported by TUBITAK grant no. 114F379.

\section{REFERENCES}

[1] T. Takan, V. A. Özkan, F. İdikut, İ. O. Yldrm, A. B. Şahin and H. Altan, "Compressive sensing imaging through a drywall barrier at sub-THz and THz frequencies in transmission and reflection modes", Proc. SPIE 9244, Image and Signal Processing for Remote Sensing XX, 92441N, 2014.

[2] N. Kakenov, T. Takan, V. A. Özkan, O. Balcı, E. O. Polat, H. Altan, and C. Kocabas, "Graphene-enabled electrically controlled terahertz spatial light modulators," Opt. Lett. 40, 2015.

[3] L. I. Rudin, S. Osher and E. Fatemi, "Nonlinear total variation based noise removal algorithms", Phys. D 60, 259, 1992.

[4] W. L. Chan, K. Charan, D. Takhar, K. F. Kelly, R. G. Baraniuk and D. M. Mittleman, "A single-pixel terahertz imaging system based on compressed sensing Applied Physics Letters 93, 121105, 2008. 